\title{
Coulisses
}

Revue de théâtre

10 | Printemps 1994

Varia

\section{Jean-Louis Barrault et Paul Claudel entre la lyrique et le mime, un dialogue difficile.}

Jacques Houriez

\section{(2) OpenEdition}

1 Journals

Édition électronique

URL : http://journals.openedition.org/coulisses/3035

DOI : $10.4000 /$ coulisses.3035

ISSN : 2546-9460

Éditeur

Presses universitaires de Franche-Comté

Édition imprimée

Date de publication : 1 juin 1994

Pagination : 68-71

ISSN : 1150-594X

\section{Référence électronique}

Jacques Houriez, « Jean-Louis Barrault et Paul Claudel entre la lyrique et le mime, un dialogue difficile. », Coulisses [En ligne], 10 | Printemps 1994, mis en ligne le 15 mars 2019, consulté le 24 octobre 2019. URL : http://journals.openedition.org/coulisses/3035; DOI : 10.4000/coulisses.3035

Ce document a été généré automatiquement le 24 octobre 2019

Coulisses 


\title{
Jean-Louis Barrault et Paul Claudel entre la lyrique et le mime, un dialogue difficile.
}

\author{
Jacques Houriez
}

1 Jean-Louis Barrault a laissé dans notre théâtre le vide créé par le grand vent de sa jeunesse enthousiaste et de sa tendresse. Il a su mettre en scène le lyrisme et l'abondance verbale de Rabelais, Shakespeare et Claudel aussi bien qu'exprimer la sentimentalité du mime des Enfants du paradis.

2 C'est ainsi qu'il a incarné dans le théâtre le visage de la tradition aussi bien que celui de l'innovation. Il a voulu porter à sa perfection tous les modes d'expression, tant celui, lyrique, du verbe, de la parole qui s'épanche, du discours qui impose la pensée que celui du geste qui exprime l'émotion. Il a été attiré par les théâtres de l'expression violente et du flot verbal. Il a appelé de ses vœux une dramaturgie qui réunisse tous les langages et s'est ainsi cru, après sa rencontre avec son dramaturge préféré en parfaite communion d'esprit avec lui : «Je crois avec Claudel que les cris, les respirations, les gestes, les mimes doivent participer avec la musique, le chant et le dialogue parlé à ce théâtre total. »

Ce n'a pas été, néanmoins sans quelque méprise. Claudel, comme Genêt qui admirait le soin avec lequel Barrault grimait la main qui mettait le feu aux orangers des colons dans Les Paravents, était fasciné par le jeu de cette même main dans Partage de midi : «Et en effet dans cette vaste cavité obscure du théâtre on ne voit plus que cette main lumineuse, tendue... vers le ciel... Éclairée par un rayon de lune $»^{1}$. Cela suggère que pour l'un et pour l'autre, malgré l'importance que revêt la parole dans leur théâtre, son langage relève essentiellement de la mimique. C'est le geste qui donne le sens. Et, de fait, lorsque, dans la même lettre, Claudel loue ou conseille, c'est la mimique qu'il désigne: «Barrault s'est souvenu de ces mains aux cent doigts jadis de Nijinsky [...] J'imagine que $\mathrm{Y}$ [sé] prend $\mathrm{M}$ [esa] par les mains et le fait tourner comme pour le regarder et se montrer elle-même sous tous les éclairages [...] $»^{2}$. Ainsi, ce que le vieux dramaturge admirait le plus dans le jeune acteur était l'héritage du mime des Enfants du 
Paradis. Il était sensible à sa capacité de transmettre l'émotion. Mais sur ce dernier point, précisément, les deux hommes divergeaient. Claudel s'était peu à peu écarté du lyrisme de ses premiers drames auquel Barrault demeurait obstinément fidèle. À ses yeux, le lyrisme, comme les grands effets, est froid et brise l'émotion. Il interdit de demeurer en contact avec l'humble humanité, ce qui est essentiel au théâtre: "En revanche, écrit-il le 18 octobre 1949 à J.-Louis Barrault qu'il vient de féliciter encore pour son jeu de mains, je n'ai pas du tout aimé cette apothéose de la fin avec cet éclairage à la Loïe Fuller et cette tirade déclamatoire continue à me donner la petite mort. Pascal a raison, en art on a toujours tort de ne pas rester en contact avec l'humble humanité. » Et il ajoute en post-scriptum :

Ce que j'aime dans le "chef-d'œuvre » ce sont les scènes jouées sans paroles en pantomime remplaçant tout ce baragouin périmé. ${ }^{3}$

4 Ce «baragouin périmé » est souvent ce que l'on apprécie le plus chez Claudel, ce qui fait préférer ses premiers drames à ceux de la maturité, ce que J.-Louis Barrault refusait de sacrifier dans les premières versions de L'Échange et du Partage. C'est aussi ce qui a conduit l'auteur à refuser toute représentation de Tête d'Or de son vivant, après l'échec d'une troisième version éliminant le lyrisme.

5 L'affrontement fut sévère. Claudel avait récrit des secondes versions supposant une mise en scène fondée essentiellement sur le gestuel. Il voulait ainsi que, dans celle de L'Échange, Marthe fût la seule femme vivante entourée de trois marionnettes. Cela supposait de la part des acteurs qui les incarnaient un jeu à la fois mécanique et mimique. Or, Barrault n'admet pas cette évolution. Il refuse les suppressions de prologue ou de finale lyriques que désire Claudel. Il obtient d'ailleurs en grande partie satisfaction, et ce sont des versions pour la scène, en quelque sorte intermédiaires qui sont jouées.

6 D'autres divergences, plus discrètes certes, mais importantes néanmoins, montrent la fidélité de Barrault à un jeu relativement traditionnel. Certes, Claudel n'envisage pas celui, codé, du théâtre balinais qu'Antonin Artaud a commenté dans Le Théâtre et son double. Cependant, pour lui, si on peut, si on doit exiger de l'acteur qu'il soit un inspiré, on ne saurait admettre pour autant qu'il s'abandonne aux hasards de l'inspiration. Lechy Elbernon, dans la seconde version de L'Échange joue «avec furie avec art». Le paradoxe de l'acteur est qu'il doit se déchaîner dans une sorte de fureur tout en restant totalement maitre de soi. Comme le futur acteur de Genêt, celui de Claudel fait participer le corps tout entier à l'expression du geste, et par là même à celle du sens. Mais il doit le faire selon une procédure aussi fixe et établie que celle de la parole. Ainsi, dans son Journal, Claudel reproche-t-il à J.-Louis Barrault un certain manque de rigueur: " [...] il n'a pas réfléchi au sens de chaque mouvement et il se livre au petit bonheur à l'inspiration q[ui] aboutit la plupart du temps à la trépidation comédienne des avant-bras, cette fois élargie à tout le corps. » Dans la mesure où le geste délivre un sens précis, il doit être parfaitement contrôlé, voire programmé.

7 Ainsi le jeu de Barrault insiste-t-il sur la spontanéité, la libre expression qui suppose une large part d'inspiration. Le jeu demandé par Claudel est tout différent. L'acteur doit se trouver dans une sorte d'état second, de transe qui lui permette d'être à la fois en soi et hors de soi. Il n'a pas le droit de s'exprimer comme il le fait en réalité dans l'improvisation du geste de Barrault. Il traduit une tout autre réalité, celle d'un personnage scénique qui n'a rien à voir avec lui-même. Jusque dans son jeu mimique, 
Barrault se veut lyrique, alors que Claudel, sans l'interdire, car il a laissé à son acteur une très grande liberté, le regrette.

8 La rencontre de l'acteur encore jeune et du dramaturge au crépuscule de sa vie fut donc profondément intime, ce fut la rencontre de deux âmes et de deux génies. Elle ne fut pas exempte cependant de difficultés, voire d'affrontements, car elle reposait, pour une grande part, sur un malentendu. Barrault recherchait en Claudel le juvénile auteur de Tête d'Or que le dramaturge avait définitivement répudié. Par sa sensibilité, sa sentimentalité même, sa conception de l'art dramatique, Barrault était demeuré un homme du début du siècle. Claudel n'a pas mis en forme sa dramaturgie. Mais les dialogues de son théâtre, ses correspondances, celle avec Barrault notamment, l'explicitent clairement. C'est véritablement une dramaturgie d'avant-garde dans laquelle les metteurs en scène de l'actuelle jeune génération n'ont pas fini de trouver un guide et une source d'inspiration.

Paul Claudel au cours d'une répétition de Partage de midi au Théâtre Marigny (1948)

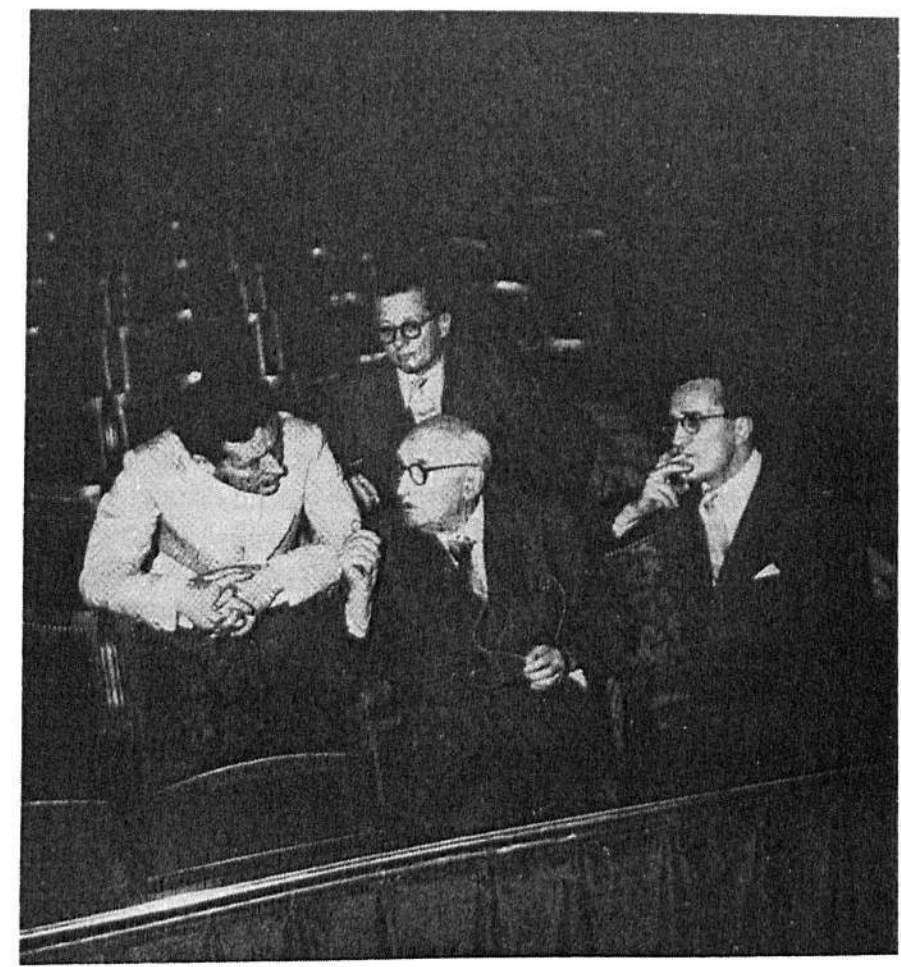

Photo Bernand

\section{NOTES}

1. Correspondance Paul Claudel - Jean-Louis Barrault, CPC 10, lettre 195, [28 février 1954], p. 267.

2. Ibidem, p. 265. 
3. Ibidem, lettre 136, p. 210 